$\begin{array}{ll}\text { le portiQue } & \text { Le Portique } \\ \text { Revue de philosophie et de sciences humaines }\end{array}$

45-46 | 2021

Art et politique, une approche critique

\title{
Présentation du texte de Varlam Chalamov
}

\section{Sergueï Soloviov}

\section{OpenEdition}

\section{Journals}

\section{Édition électronique}

URL : http://journals.openedition.org/leportique/3708

DOI : $10.4000 /$ leportique.3708

ISSN : $1777-5280$

\section{Éditeur}

Association "Les Amis du Portique"

\section{Édition imprimée}

Date de publication : 10 janvier 2021

Pagination : 19-22

ISBN : 978-2-916332-48-2

ISSN : 1283-8594

\section{Référence électronique}

Sergueï Soloviov, «Présentation du texte de Varlam Chalamov », Le Portique [En ligne], 45-46 | 2021, document 1, mis en ligne le 10 mars 2021, consulté le 26 mars 2021. URL : http://

journals.openedition.org/leportique/3708; DOI : https://doi.org/10.4000/leportique.3708

Ce document a été généré automatiquement le 26 mars 2021.

Tous droits réservés 


\title{
Présentation du texte de Varlam Chalamov
}

\author{
Sergueï Soloviov
}

1 Le discours public le plus célèbre de Varlam Chalamov, outre une courte lecture de poésie à la télévision en mai 1962 dont l'enregistrement n'a pas été conservé, est son intervention lors de la soirée à la mémoire d'Ossip Mandelstam en mai 1965. D'après les témoignages de la plupart des participants à cette soirée, ce fut son discours le plus impressionnant ${ }^{1}$.

2 En ce qui concerne le présent texte (rédigé pour une autre soirée, prévue l'année suivante), il faut prendre conscience du fait qu'il s'agit de la préparation d'une prise de parole, dans un style oral, qui devait commencer et se terminer par les vers d'Ossip Mandelstam. Finalement, cet événement n'a pas eu lieu, et le discours soigneusement préparé par Chalamov resta dans les archives ${ }^{2}$.

3 Ce qui attire l'attention, c'est une critique violente de la révolution, sans précédent dans l'œuvre de Chalamov, à part le brouillon d'une lettre à Nadejda Mandelstam, dans lequel il écrit que « le massacre des chats et des gens est l'une des caractéristiques du socialisme $»^{3}$. Mais cette version n'a pas été envoyée, et la version finale, préservée dans les archives de Nadejda Mandelstam, est beaucoup plus laconique et ne contient pas de telles phrases". Par ailleurs, il est évident que par "socialisme », Chalamov n'entendait pas la doctrine ou l'idéal social, mais le « socialisme réel »- le système soviétique dans sa version stalinienne et néo-stalinienne. À cette époque-là, ainsi que plus tard, Chalamov n'a pas renoncé à sa plus haute appréciation de la tradition révolutionnaire, des membres du mouvement Narodnaïa Volia et du Parti socialiste révolutionnaire ; dans les années 1970 il écrivait notamment des poèmes dédiés à Che Guevara et un essai sur le révolutionnaire bolchévique Fiodor Raskolnikov ${ }^{5}$. Il est important aussi de tenir compte du fait qu'après être rentré à Moscou, Chalamov avait grand besoin d'un interlocuteur avec qui il serait sur un pied d'égalité, et cherchait des lecteurs et amis avec lesquels il pourrait parler la même langue. Dans cette situation, il était prêt à faire des compromis - dans une mesure limitée - pour s'adapter un peu à des interlocuteurs qu'il appréciait. Cela se révèle clairement dans sa correspondance 
avec Boris Pasternak, Natalia Stoliarova et surtout Nadejda Mandelstam. Certes, dans les années 1965-1966, son jugement sévère sur la révolution a pu être affecté par la tristesse de la perte d'un chat adoré, ainsi que par les faits traumatisants qu'il évoque dans son texte.

L'année 1966 est peut-être la période de son rapprochement le plus étroit avec Nadejda Mandelstam et son entourage. La même année, Chalamov a remis un manuscrit des Récits de la Kolyma au spécialiste de langue slave Clarence Brown, qu'il avait rencontré chez Nadejda Mandelstam ${ }^{6}$. La même année, Chalamov en ayant été informé, Les Récits de la Kolyma furent diffusés par le samizdat ${ }^{7}$. Enfin, c'est à cette époque que Chalamov rédigea la Lettre à un vieil ami, qui fut ensuite publiée par le célèbre dissident Alexandre Ginsbourg dans le Livre blanc, compilé à partir des documents du procès de Siniavski et Daniel. Il est fort probable que le texte présenté ci-dessous, ainsi que la Lettre à un vieil ami aient été rédigés en parallèle, ou immédiatement l'un après l'autre.

Destinés à un large public, ces deux textes ont été écrits à une époque où Chalamov était au plus près des milieux dissidents. En 1965, il participa à une manifestation sur la place Pouchkine à Moscou en soutien à Siniavski et Daniel, et il apprécia hautement que cette manifestation eût lieu. La période du " dégel » qui, contrairement à l'opinion répandue, ne s'était pas terminée en 1964 avec la destitution de Nikita Khrouchtchev du poste de Premier secrétaire du Comité central du Parti communiste de l'Union soviétique, atteignait sa fin. Un des signes les plus évidents de la fin de cette époque fut le renforcement de la censure. Ce texte témoigne du fait qu'aux yeux de Chalamov, le resserrement de la censure était horrible. Lorsqu'il écrit avec fureur sur le fait que Mandelstam n'est pas publié, il écrit aussi sur lui-même. Non seulement il avait été mourant à la Kolyma et s'était retrouvé à Vladivostok un an avant Mandelstam, mais il avait aussi été étouffé par l'impossibilité d'avoir un public. Cette pensée résonne aussi dans la Lettre à un vieil ami :

«Chaque écrivain veut être publié. Le tribunal ne peut-il vraiment pas comprendre qu'un écrivain a besoin de la publication comme de l'air?

Combien sont morts parmi ceux qui n'étaient pas autorisés à publier leurs œuvres? Qu'est devenu Le Docteur Jivago de Pasternak ? Qu'est devenu Platonov ? Qu'est devenu Boulgakov? La moitié des œuvres de Boulgakov et le quart de tout ce que Platonov a écrit ont été publiés. Mais ce sont les meilleurs écrivains en Russie. Autrefois, il suffisait de mourir pour qu'on publiât quelque chose, mais Mandelstam est privé même de ce destin. $»^{8}$

6 Comme Mandelstam, Chalamov a été aussi privé de publication pendant longtemps, tout en étant parfaitement conscient de son génie : Mandelstam avait compris qu'il était le " plus grand poète de Russie ", Chalamov pensait que les Récits de la Kolyma étaient parfaits, il était également conscient qu'il restait privé de lecteur.

En 1968, Chalamov rompit ses relations avec Nadejda Mandelstam et son entourage, et peu après, ses jugements sur le mouvement de dissidence devinrent extrêmement défavorables ${ }^{9}$. Pourtant, son respect à l'égard du rôle joué par Mandelstam dans la culture russe, ainsi que son sentiment envers les contraintes de la censure, ne changèrent pas. 


\section{NOTES}

1. P. M. Nerler, «Une soirée commémorative à Mandelstam à la Faculté de mécaniques et de mathématiques (1965) : reconstitutions ", in Racines, pousses, fruits... : Les Journées de Mandelstam à Varsovie, Tome 2, Les Éditions de RGGU, Moscou, 2015, p. 587-616 (en russe).

2. En plus du texte final, il y a 43 feuilles de brouillons inédits aux Archives d'État de la littérature et de l'art (RGALI), fond 2596, inventaire 2, document 123.

3. Varlam Chalamov, Euvres complètes en 6 tomes et 1 tome complémentaire, tome 6, Moscou, Les Éditions de Knijniï Kloub Knigovek, 2013, p. 418-419.

4. Archives d'État de la littérature et de l'art (RGALI), fond 1893, inventaire 3, document 273, feuilles 5-8.

5. Cf. Sergueï Soloviev, « Une inévitable solitude : Varlam Chalamov et la tradition idéologique », in Mémoires En Jeu, nº1, 2016, p. 68-75 (version originale en langue russe publiée en 2012).

6. Cela a été définitivement démontré par Yacha Klots dans un article où il restitue en détail l'histoire de la relation entre Chalamov et la presse des émigrés russes. Cf. Yacha Klots, " Chalamov vu par l'émigration russe. Les Récits de la Kolyma dans le Novy Journal » in "La loi de la résistance à la décomposition... " La spécificité de la prose et de la poésie de Varlam Chalamov et leur perception au début du XXI siècle. Recueil d'articles scientifiques, éd. Loukach Babka, Sergueï Soloviev, Valériï Yessipov, Jan Makhonin, 2017, Prague-Moscou, p. 231-257 (en russe).

7. Auto-édition en russe. Il s'agit d'un système clandestin de production et de circulation d'écrits en dehors des éditions d'État durant l'époque soviétique [ndt].

8. Varlam Chalamov «Lettre à un vieil ami » in Le prix d'une métaphore, ou Crime et châtiment de Siniavski et Daniel, éd. E. M. Velikanova, Les Éditions de Kniga, Moscou, 1989, p. 524 (en russe).

9. Valérii Yésipov, Chalamov, Les Éditions de Molodaïa gvardia, Moscou, 2012, p. 275-280 (en russe).

\section{AUTEUR}

\section{SERGUEİ SOLOVIOV}

Sergueï Soloviov, docteur en philosophie de l'Université d'État Pédagogique de Moscou, est chercheur aux Archives d'État russe de l'histoire socio-politique, ainsi qu'à la Faculté des Sciences Politiques de l'Université d'État de Moscou. Il est l'un des créateurs du projet Shalamov.ru, qu'il dirigea de 2008 à 2019, actuellement rédacteur de la revue en ligne Skepsis. 